\title{
Propuesta de caracterización jerárquica de los ríos españoles para su clasificación según la Directiva Marco de la Unión Europea
}

\author{
Marta González del Tánago y Diego García de Jalón
}

E.T.S. Ingenieros de Montes, Universidad Politécnica de Madrid, Ciudad Universitaria, 28040-Madrid. marta.gtanago@upm.es.diego.gjalon@upm.es.

\section{RESUMEN \\ Propuesta de caracterización jerárquica de los ríos españoles para su clasificación según la Directiva Marco de la Unión Europea}

La clasificación de los ríos tiene un papel importante en el manejo y conservación de los ecosistemas fluviales, y representa una fase inicial en la implementación de la Directiva Marco del Agua. Según han ido evolucionando los conocimientos científicos de la ecología fluvial y las técnicas informáticas para la caracterización espacial de las regiones hidrográficas, han ido surgiendo diferentes sistemas de clasificación taxonómica y de regionalización del territorio, integrando finalmente el río en su cuenca vertiente.

En este trabajo se presenta una revisión histórica de los principales sistemas de clasificación de los ríos, y se propone un esquema de caracterización jerárquica donde se contemplan diferentes escalas, de provincia biogeográfica, cuenca vertiente, segmento fluvial y tipo de hábitat, atendiendo a los principales factores que determinan las comunidades biológicas de los ecosistemas fluviales.

El esquema de caracterización propuesto está diseñado para ser utilizado mediante sistemas de información geográfica, a partir de cartografías y de un trabajo de campo para la escala de hábitat fluvial. Es un método de caracterización abierto, de tipo arborescente, que admite la incorporación de nueva información y nuevas características y subclases en cada una de las escalas consideradas, permitiendo establecer un esquema de clasificación con base hidrológica y ecológica común para todo el territorio español, a la vez que contener información particularizada para las distintas regiones hidrográficas. Incluye todos los factores obligatorios de clasificación según la Directiva Marco, así como algunos de los elementos hidromorfológicos que establece la Directiva, para identificar el estado ecológico de los distintos tramos fluviales y proponer medidas para su gestión (restauración) y conservación.

Palabras clave: Ríos, Caracterización, Clasificación, Tipología, Directiva Marco del Agua, clasificación jerárquica, clasificación geomorfológica.

\footnotetext{
ABSTRACT

A proposal of hierarchic characterization of the Spanish rivers for their classification in accordance to the Water Framework Directive of the European Union

River classification has an important role in the management and conservation of fluvial ecosystems, and represents a first step in the implementation of the European Water Framework Directive. According to the development of scientific knowledge in fluvial ecology and computer techniques for the spatial characterisation of hydrographic regions, different systems of taxonomic and regional classification have been proposed, finally integrating the river within the context of its watershed. In this paper, the historical evolution of river characterisation schemes is reviewed, and a hierarchical characterisation approach is proposed according to different scales, biogeographic provinces, watershed, river segments, and river reaches attending to the main factors determining biological communities in river ecosystems.

This proposed characterisation scheme is prepared to be used by GIS, from maps and fieldwork at the reach scale. It is an open-tree characterisation method, where new information can be incorporated and some of the criteria can be further detailed, adding new characteristics and subclasses at the different scales being considered, allowing for the development of a classification scheme based on hydrological and ecological criteria in common for the whole Spanish territory. It includes all the mandatory factors for river classification according to the Water Framework Directive, as well as some of the hydromorphological elements established by the Directive for identifying the ecological status of the different fluvial stretches, and for proposing management (restoration) and conservation measures.
} 
Keywords: Rivers, Characterisation, Typology, Water Framework Directive, Hierarchical classification, Geomorphological classification.

\section{INTRODUCCIÓN}

La acción de clasificar tiene un papel muy importante en el desarrollo científico, ya que permite ordenar las observaciones y toma de datos de campo, facilita la interpretación de las formas y procesos analizados, e induce a la formulación de leyes empíricas y teorías que expliquen las diferencias de la estructura y del funcionamiento de los objetos clasificados (Goodwin, 1999).

En relación a los ríos, su clasificación es una tarea esencial para interpretar la distribución de los distintos tipos de funcionamiento ecológico en sus respectivas cuencas vertientes, e indispensable para poder desarrollar estrategias de gestión y conservación, de acuerdo a las diferentes pautas de funcionamiento ecológico (Naiman, 1998).

Esta importancia de la clasificación de los ríos en su gestión y conservación ha sido reconocida en la Directiva Marco del Agua (Comisión Europea, 2000), donde se requiere a los Estados de la Comunidad Europea una tipificación inicial de sus masas de agua, sobre la que se puedan definir las condiciones específicas de referencia de cada tipo de río, llevar a cabo la evaluación del estado ecológico, y establecer medidas de prevención de la degradación y protección y mejora de su estado actual.

En este trabajo se propone un sistema de caracterización de los ríos españoles basado en criterios hidromorfológicos y ecológicos, válido para su clasificación atendiendo a los objetivos de la Directiva Marco. Se trata de un esquema de caracterización jerárquica a diferentes escalas, que incluye los conceptos propuestos por diferentes autores en el ámbito de la integración del río y su cuenca vertiente, sobre los que se presenta una revisión histórica detallada, la cual abarca también algunas propuestas recientes de clasificación de ríos realizadas en el mismo contexto de la Directiva Marco.

\section{ANTECEDENTES}

\section{Reseña histórica}

Son muchos los sistemas de clasificación de los ríos que se han venido proponiendo desde que se inició su estudio científico, atendiendo a diferentes objetivos y considerando distintos criterios y enfoques de clasificación. Diferentes autores han elaborado revisiones bibliográficas del tema (Naiman et al. 1992; Rosgen, 1994; Thorne, 1997; Kondolf et al., 2003, etc.), permitiendo analizar el desarrollo del conocimiento científico de los ecosistemas fluviales, y la influencia que ha tenido en dicho desarrollo la evolución de las técnicas informáticas, facilitando los estudios territoriales de los ríos integrados en sus respectivas cuencas vertientes.

En el trabajo de Bailey et al. (1978) se describen los diferentes métodos que pueden utilizarse para la clasificación de los sistemas naturales, todos ellos útiles según los objetivos propuestos, diferenciando las clasificaciones taxonómicas (clasificación de localidades definidas por una serie de atributos), de las clasificaciones de un territorio mediante mapas sucesivos ("regionalización"), en los que se reconocen áreas donde existe una cierta homogeneidad interna respecto a ciertas características, que contrasta con la de las zonas adyacentes.

En relación a las clasificaciones taxonómicas, éstas pueden referirse a atributos que respondan a uno o a varios factores del ecosistema fluvial (clasificaciones basadas en variables biológicas, geomorfológicas, del régimen de caudales, etc.) y pueden estar basadas o no en los procesos físicos y ecológicos que rigen el funcionamiento de los ecosistemas acuáticos, así como atender a una o a varias escalas, pudiendo ser establecidas a su vez mediante enfoques deterministas o estadísticos, de carácter agregativo o divisivo.

Las clasificaciones basadas en la regionaliza- 


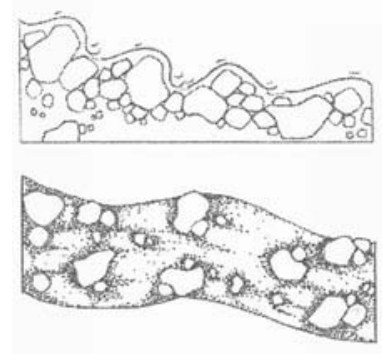

a) Cascadas
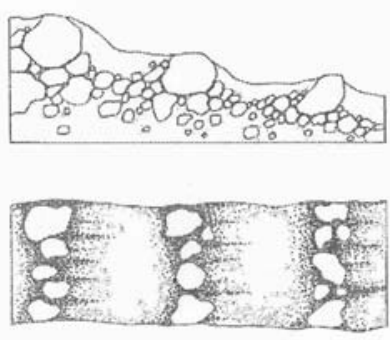

b) Saltos y pozas

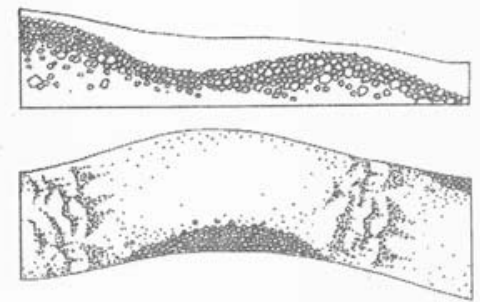

c) Rápidos y remansos

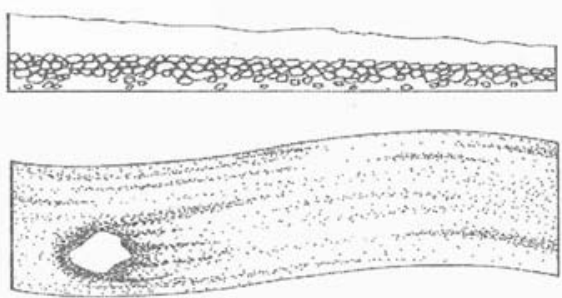

d) Rápido continuo (tabla)
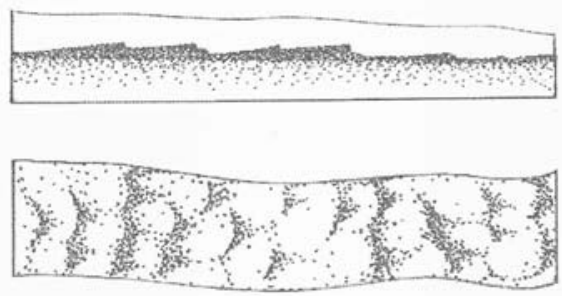

e) Dunas y rizaduras

Figura 1. Tipos morfológicos del substrato del cauce definidos por Montgomery \& Buffington (1993), relacionados con el tamaño de los sedimentos y su disposición sobre el lecho del río. Morphological types of river substratum defined by Montgomery \& Buffington (1993), related to sediment size and their arrangement in the riverbed.

ción del territorio responden siempre a un enfoque con fundamento físico, que atiende a las variables que van determinando las diferencias observadas a diferentes escalas espaciales y temporales, y generalmente son de tipo jerárquico, multiescalar y de carácter divisivo.

Los diferentes métodos propuestos para la clasificación de los ríos responden a estos dos esquemas metodológicos. Inicialmente predominan los enfoques taxonómicos, donde la caracterización de los distintos tramos fluviales es unidisciplinar, reflejando aspectos parciales del ecosistema fluvial, según el campo de especialidad de sus respectivos autores; y posteriormente, según avanza el conocimiento científico de los ríos y los sistemas de información geográfica, se desarrollan las clasificaciones basadas en la regionalización del territorio, atendiendo a una visión más integradora y multidisciplinar, donde se relacionan las características del tramo fluvial con las de su entorno físico, atendiendo a los diferentes procesos físicos y ecológicos que intervienen.

\section{Clasificaciones taxonómicas de los tramos fluviales}

En el ámbito de la ecología fluvial, los primeros intentos de clasificación de los ríos atienden a sus características biológicas, relativas a las especies piscícolas (Huet, 1954), a los grupos de invertebrados (Illies \& Botosaneanu, 1963; Wright et al., 1989) o a las plantas acuáticas (Holmes, 1989), presentando en muchos casos una interpretación del funcionamiento del sistema fluvial (Cummins, 1974), y la relación entre las especies indicadoras utilizadas en la clasificación, y los factores físicos implícitos que determinan la zonación de los sucesivos hábitats fluviales (Hawkes, 1975).

Posteriormente, siguiendo este esquema de clasificación otros autores han diferenciado tipos de ríos atendiendo a la importancia relativa de los principales cationes y aniones constituyentes de la calidad de sus aguas (Meybeck $\&$ Helmer, 1989), o a las diferencias en la varia- 
bilidad y predictibilidad de su régimen de caudales (Poff \& Ward, 1989), relacionando dichas características con la estructura de las comunidades biológicas, y la capacidad de recuperación frente a perturbaciones naturales o antrópicas de los sistemas fluviales (Poff \& Ward, 1990).

En el campo de la geomorfología fluvial, los trabajos iniciales de clasificación también son de tipo taxonómico, centrándose en el reconocimiento de las diferentes formas del trazado de los ríos. Leopold \& Wolman (1957) diferencian tres tipos de ríos, rectos, meandriformes y trenzados, proponiendo unos valores umbrales de caudal y pendiente longitudinal para predecir la respuesta geomorfológica a cambios en estas variables, y posteriormente Brice (1975) describe también el trazado en planta de los ríos atendiendo a su grado de sinuosidad, diferenciando las formas trenzadas y anastomosadas.

Schumm (1977) resalta la influencia de la dinámica fluvial, y diferencia los ríos controlados por afloramientos rocosos que determinan su morfología, de los ríos aluviales, libres para ajustar sus dimensiones y formas, entre los que distingue los ríos estables, de los ríos con predominio de erosión o de sedimentación.

Más recientemente, Montgomery \& Buffington (1993) proponen una clasificación de los ríos de la costa pacífica de Washington atendiendo a los procesos fluviales que intervienen a diferentes escalas espaciales. Estos autores definen en estos ríos de montaña siete tipos de tramos fluviales, diferenciando por una parte los de origen coluvial o confinados por afloramientos rocosos, de los de origen aluvial, diferenciando entre estos últimos, cinco tipos morfológicos distintos según la estructura del substrato, en un esquema de zonación altitudinal siguiendo el gradiente de la pendiente longitudinal y los procesos fluviales asociados (Fig. 1). Adicionalmente, también definen los tipos de ríos cuya morfología queda forzada por la presencia de restos vegetales.

Finalmente, Rosgen (1994, 1996) propone una clasificación de los ríos basada en diferentes variables y niveles jerárquicos, que ha tenido una gran aceptación en el ámbito de la restauración fluvial entre los servicios forestales de Estados Unidos, así como una cierta crítica por parte de diferentes autores (Kondolf, 1995; Doyle et $a l ., 1999)$. En un primer nivel de clasificación, Rosgen diferencia nueve tipos de ríos, de acuerdo con su pendiente longitudinal, sinuosidad y forma de la sección transversal (Fig. 2). En un segundo nivel de clasificación, cada uno de estos siete tipos de ríos, denominados con letras mayúsculas (A, A+, B, C, D, DA, E, F y G) se subdivide a su vez según su rango de pendiente y granulometría del substrato, obteniendo 42 subtipos, denominados con números y letras minúsculas (A1, A2, B1b, B2c, etc). Un tercer nivel de clasificación alude al estado ecológico del río, descrito en base a su vegetación riparia, fauna acuática, régimen de caudales, estabilidad de sus orillas, etc., existiendo un cuarto nivel para verificar y predecir ciertas variables hidráulicas y de transporte de sedimentos.

\section{Regionalización del territorio: Clasificación de los ríos en el contexto de su cuenca vertien}

En 1975, Hynes propone un enfoque integrado del río y su entorno, describiendo de forma pionera las relaciones existentes entre los procesos hidrológicos del valle y las características y funcionamiento del ecosistema fluvial.

Los nuevos conceptos relacionando el río con su cuenca vertiente, surgidos en base a toda una línea de investigación hidrológica en cuencas experimentales (Douglass \& Hoover, 1988), facilitan la propuesta de nuevos enfoques de clasificación de los ríos, que integran a los sistemas acuáticos en sus sistemas terrestres circundantes.

Los trabajos de Platts (1974) relacionando las condiciones geomórficas de los ríos con sus poblaciones de salmónidos, y los de Likens et al. (1977) o Borman \& Likens (1981) proponiendo las cuencas vertientes pequeñas (watersheds) como ecosistemas para el estudio de los ciclos de nutrientes y procesos hidrológicos, constituyen antecedentes fundamentales para establecer el marco conceptual propuesto por Warren (1979) y Lotspeich (1980), en el que consideran la cuenca vertiente como ecosistema básico de clasificación natural, y Lotspeich \& Platts (1982) desarrollan el sistema integrado de 


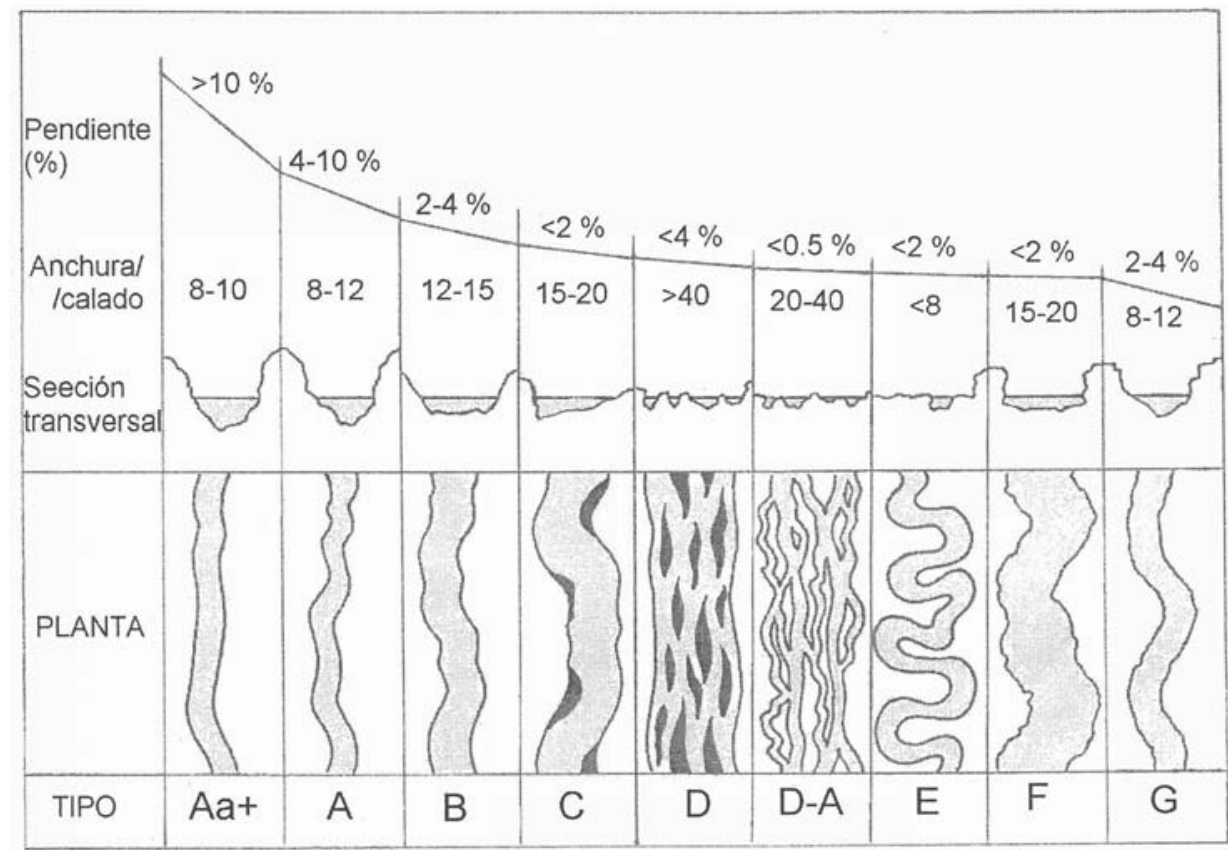

Figura 2. Tipos morfológicos de ríos diferenciados por Rosgen (1996) en el Nivel I de su clasificación. River morphological types identified by Rosgen (1996) at the Level I in his classification.

clasificación para la gestión de los ecosistemas acuáticos y su fauna piscícola.

En el escenario científico generado por todos estos conceptos, Frissell et al. (1986) proponen un sistema de clasificación jerárquica de los ríos integrados en su cuenca vertiente, asumiendo un determinismo biológico respecto a los factores físicos del hábitat, los cuales vienen a su vez condicionados sucesivamente, y de forma ascendente, por las características del tramo de río, perteneciente a un segmento fluvial, que forma parte de una red de drenaje, que a su vez depende de su cuenca vertiente. Una vez propuesta esta organización jerárquica, atendiendo a diferentes escalas geográficas, dichos autores acotan espacialmente los bordes o límites entre escalas, y clasifican los distintos tipos de hábitats que pueden existir en cada caso. En este sentido reconocen una "organización" de la heterogeneidad de los hábitats en el contínuo fluvial de Vannote et al. (1980), y consideran la estructura, el desarrollo y la persistencia de cada hábitat como factores ligados al funcionamiento hidrológico de la cuenca vertiente, con los cuales se puede predecir su capacidad o potencial para albergar a las diferentes especies.

Este enfoque integrado de organización jerárquica de los hábitats fluviales propuesto por Frissell et al. (1986) ha sido asimilado en posteriores sistemas de clasificación de los ríos basados en procesos físicos (Montgomery \& Buffington, 1997), sirviendo a partir de entonces de marco conceptual básico para el desarrollo de estrategias de gestión y restauración fluvial (Doppelt et al., 1993; Williams et al.1997, etc.).

Paralelamente a estos trabajos, y con la finalidad de facilitar la gestión y planificación de uso múltiple en los espacios naturales, se desarrollan por parte de los Servicios Forestales de Estados Unidos diferentes propuestas de clasificación de usos y suelos, teniendo en cuenta las posibilidades que se vislumbran en este campo con el desarrollo de la recogida de información vía satélite, y de las técnicas informáticas para su análisis (Driscoll et al., 1978), surgiendo una necesidad de unificar criterios (Nelson et al., 1978), atendiendo a los diferentes objetivos de planificación. 
Este enfoque de clasificación del territorio es utilizado para definir las ecoregiones de Estados Unidos, primero por Bailey (1976), el cual propone un sistema de regionalización jerárquica, atendiendo sucesivamente a la influencia del clima, la vegetación natural potencial, los suelos y la formas del relieve; y posteriormente por Omernik (1987), éste último con una finalidad enfocada a la gestión de la calidad de las aguas de los ecosistemas acuáticos, haciendo especial hincapié en la influencia del uso del suelo.

Las diferentes propuestas de ecoregiones son contrastadas posteriormente por sus respectivos autores (Bailey, 1983; 1995; Omernik 1995), integrando en sucesivas publicaciones los conceptos del río y su cuenca vertiente, en un territorio configurado por diferentes factores, que inciden a distinta escala espacial y temporal (Omernik \& Bailey, 1997; Griffith et al., 1999).

Más recientemente, la necesidad de generalizar los sistemas de gestión y planificación de los ecosistemas acuáticos, junto al desarrollo de los sistemas de información geográfica y la disponibilidad de cartografía digitalizada para el estudio de grandes cuencas, ha propiciado el desarrollo de numerosos estudios, donde se analiza la relación entre las distintas clasificaciones del territorio según ecoregiones y cuencas vertientes, y las características biológicas de los respectivos ecosistemas acuáticos (Hawkins \& Norris, 2000).

En este sentido, (Hawkins et al., 2000) llegan a la conclusión de que las regionalizaciones a gran escala (ej. ecoregiones) absorben una parte relativamente pequeña de la variabilidad biológica y son de uso limitado en el campo de los indicadores biológicos, como ya destacaron en su día Omernik \& Bailey (1997), mientras que tienen un interés notable en la estratificación inicial del territorio para seleccionar las localidades de estudio a pequeña escala, asegurando una representación completa de las distintas condiciones ambientales existentes.

Hoy día es indudable el interés de utilizar regionalizaciones del territorio a diferentes escalas para la clasificación de los ecosistemas acuáticos, dada la facilidad de disponer de información y herramientas adecuadas para ello, que permiten reconocer los distintos flujos y dimensiones del
Tabla 1. Sistema A de clasificación de los ríos según la Directiva Marco del Agua. System A for river classification according to the Water Framework Directive.

\begin{tabular}{ll}
\hline Tipología & \multicolumn{1}{c}{ Descriptores } \\
\hline Ecoregion & Ecoregiones (ver mapa A en Annexo XI) \\
Tipo & Altitud \\
& - Zonas Altas: $>800 \mathrm{~m}$ \\
& - Altitud Media: 200 to $800 \mathrm{~m}$ \\
& Tamaño de la cuenca vertiente \\
& - Pequeña: 10 a $100 \mathrm{~km}^{2}$ \\
& - Media: $>100$ a $1000 \mathrm{~km}^{2}$ \\
& - Grande: $>1000$ a $10000 \mathrm{~km}^{2}$ \\
& - Muy grande: $>10000 \mathrm{~km}^{2}$ \\
& Geología \\
& - Caliza \\
& - Silícea \\
& Orgánica \\
\hline
\end{tabular}

sistema fluvial (Amorós \& Petts, 1993), así como interpretar la influencia de los principales factores que determinan su funcionamiento ecológico a diferentes escalas espacio-temporales, de carácter natural o antrópico (Robertson \& Saad, 2003; Detenbeck et al., 2004).

Sin restar interés a las clasificaciones de tipo taxonómico, equivalentes a modelos de "caja negra" donde la confluencia de atributos permite agrupar a los objetos clasificados, para posteriormente interpretar los grupos o clases obtenidas, creemos que hoy día son las clasificaciones basadas en la regionalización del territorio, y en el conocimiento de la dependencia hidrológica y ecológica del río respecto a su entorno, las que permiten caracterizar con mayor base fisica y ecológica los distintos tipos de sistemas y hábitats fluviales, facilitando al mismo tiempo su gestión y planificación de acuerdo a los diferentes tipos de estructura y de funcionamiento.

\section{La clasificación de los ríos en la Directiva Marco del Agua}

La Directiva Marco del Agua establece como etapa inicial para su implementación, la clasificación de las aguas superficiales atendiendo a los siguientes tipos de masas de agua: 
- ríos

- lagos

- aguas de transición

- aguas costeras

- masas de agua artificiales o altamente modificadas.

Según esta distinción, los tramos fluviales pueden corresponder a la categoría "río" o a la de aguas de transición, masas artificiales o altamente modificadas, según sea la influencia costera y el grado de artificialidad o perturbación, respectivamente.

Refiriéndose al caso de los "ríos", la Directiva Marco propone dos sistemas de caracterización, atendiendo a diferentes variables o atributos, como se refleja en las Tablas 1 y 2.

El sistema A trata de enmarcar al río en su entorno biogeográfico, e incluye variables relacionadas con los procesos hidrológicos a escala de cuenca vertiente, permitiendo establecer una tipología regional a dicha escala, común para todo el ámbito europeo.

El sistema B propone una serie de factores opcionales para la caracterización de los ríos que actúan a diferentes escalas espaciales, de hábitat fluvial (ej. anchura y profundidad del agua, tipo de substrato), de tramo o segmento fluvial (ej. caudales líquidos y sólidos, forma del valle, trazado del cauce) o de laderas vertientes (ej. rango de temperatura del aire, precipitación), propiciando el establecimiento de una tipología taxonómica, de confluencia de atributos, no necesariamente basada en una organización jerárquica de procesos físicos y ecológicos.

El objetivo de esta clasificación es establecer una tipología de las masas de agua, que permita a cada Estado miembro establecer unas condiciones de referencia para cada tipo de río existente, en términos biológicos, hidromorfológicos y físico-químicos, con las que posteriormente se pueda evaluar el estado ecológico de los diferentes tramos fluviales, de acuerdo a la referencia del tipo al que corresponda.

Aunque se ha desarrollado una estrategia común de implementación de la Directiva Marco, y redactado documentos guías para la clasificación del estado ecológico (REFCOND,
Tabla 2. Sistema B de clasificación de los ríos según la Directiva Marco del Agua. System B for river classification according to the Water Framework Directive.

\begin{tabular}{cl}
\hline $\begin{array}{c}\text { Caracterización } \\
\text { Alternativa }\end{array}$ & $\begin{array}{l}\text { Factores físicos y químicos que } \\
\text { determinan las características del } \\
\text { río o parte del río e influyen en la } \\
\text { estructura y composición de las } \\
\text { poblaciones biológicas }\end{array}$ \\
\hline Factores obligatorios & Altitud \\
& Latitud \\
& Longitud \\
& Geología \\
& Tamaño \\
\hline Factores opcionales & Distancia la origen del río \\
& Energía hidráulica \\
& Anchura media de agua \\
& Profundidad media de agua \\
& Pendiente media del agua \\
& Forma y trazado del cauce principal \\
& Categoría del caudal \\
& Forma del valle \\
& Transporte de sedimentos \\
& Capacidad de neutralización de ácidos \\
Composición media del substrato & Cloruros \\
Rango de temperatura del aire \\
Temperatura media del aire \\
Precipitación \\
\hline
\end{tabular}

2003) y la caracterización de las condiciones hidromorfológicas (CEN, 2004), no se ha llegado a establecer una metodología de clasificación tipológica común para todo el ámbito de la Comunidad europea. De esta forma, han surgido diferentes sistemas de clasificación de los ríos, aplicados en cada Estado miembro, o incluso en cada región dentro de un mismo Estado miembro.

A modo de ejemplo, en Francia, Wasson et al. (2002) han definido una tipología de las aguas corrientes a través del reconocimiento de "hidroecoregiones". En un primer nivel de regionalización, dichos autores diferencian 22 hidroecoregiones, atendiendo a características de clima, relieve y geología; el tipo de roca es estimado a partir de determinadas variables cuantitativas relacionadas con la permeabilidad, la resistencia a la erosión y la naturaleza química de las escorrentías, las cuales permiten realizar un análisis de componentes principales, $\mathrm{y}$ formar tres grupos de hidroecoregiones en 
base a estas variables geológicas. El relieve es estimado a partir de tres variables, la altitud, la pendiente del terreno y la pendiente de la línea que une los puntos más bajos del cauce (thalweg), para las que se han definido unos rangos de valores y definido distintas clases de altitud y pendiente, respectivamente. $\mathrm{Y}$ finalmente, el clima se ha analizado a través de cuatro variables, la precipitación anual, las precipitaciones máximas en 24 horas, la variación estacional de precipitaciones y la temperatura máxima media del mes de Julio, respecto a las cuales también se han definido unas clases en relación al rango de valores absolutos y relativos de dichas variables.

En un segundo nivel de regionalización, dentro de cada una de estas 22 hidroecoregiones se ha estudiado la posibilidad de diferenciar distintas subregiones en base a condiciones locales de tipo geológico o climático, facilitando su agrupación geográfica.

En Holanda han definido una serie de "tipos de ríos" atendiendo a la pendiente del cauce y velocidad de las aguas, geología y superficie de cuenca vertiente, para cuyo reconocimiento se diseña una clave taxonómica donde aparecen sucesivamente los rangos de valores establecidos para estas variables. De forma similar, en Chipre se ha propuesto una tipología de ríos que diferencia en primer lugar el origen de las aguas y la pendiente longitudinal del cauce, posteriormente la naturaleza geológica, a continuación la superficie vertiente y la precipitación, y finalmente el tipo de régimen, permanente o efímero, considerando, a cada nivel taxonómico de clasificación, distintos rangos de valores de las variables consideradas.

En España también se han propuesto distintos métodos para la tipología de los ríos atendiendo a la Directiva Marco. Así, Bonada et al. (2002), a partir del sistema B de clasificación de la Directiva Marco, proponen una tipología de las cuencas mediterráneas analizadas en el proyecto GUADALMED en la que se diferencian 6 ecotipos, atendiendo al tamaño de río (grande, mediano, pequeño, rambla), geología de la cuenca (silícea, calcárea o sedimentaria), y posición (tramo de cabecera, medio-bajo y bajo).
La clasificación obtenida por estos autores resulta excesivamente simplificada, de tipo taxonómico, donde en cada una de las clases obtenidas puede haber una gran diversidad de condiciones ecológicas, sin que a nuestro entender pueda ser útil para establecer las condiciones de referencia, biológicas e hidromorfológicas, respectivas para cada clase establecida.

Con más precisión hidrológica, la Agencia Catalana del Agua (ACA, 2002) ha diferenciado 5 regiones fluviales y 10 subregiones de gestión fluvial, atendiendo a criterios hidrológicos y geológicos. Para su reconocimiento se establece una clave taxonómica, donde los criterios utilizados no están organizados de forma jerárquica, en base a procesos físicos o hidrológicos.

Ollero et al. (2003) han propuesto una clasificación de los ríos de Aragón atendiendo a sus características geomorfológicas, donde se consideran variables topográficas, hidroclimáticas y morfológicas, siguiendo un esquema jerárquico en el que se reconocen la escala de ecoregión (caracterizada por la altitud y la localización geográfica) y la del segmento fluvial (atendiendo a la forma del valle y el caudal específico). Esta tipología de los ríos aragoneses responde a los procesos físicos que configuran la morfología de los distintos tramos de ríos, pero no incluye algunas variables como el régimen de caudales y el tipo de substrato, que pueden influir notablemente en las características ecológicas de cada tramo.

Finalmente, el Centro de Estudios Hidrográficos (CEDEX, 2004) ha establecido una caracterización de los ríos españoles diferenciando 29 tipos ambientales, que han sido obtenidos mediante una clasificación divisiva en base al caudal medio anual, pendiente media de la cuenca, altitud, conductividad de las aguas, temperatura media anual y orden del río. Inicialmente se han considerado tres regiones distintas, la región mediterránea, la región atlántica (euosiberiana) y la región macaronésica, y en cada una de ellas se ha procedido a la clasificación divisiva de los cursos de agua atendiendo a las variables mencionadas, si bien en un orden de consideración distinto en cada una de ellas.

El análisis conjunto de los distintos sistemas propuestos hasta la fecha para la caracterización 


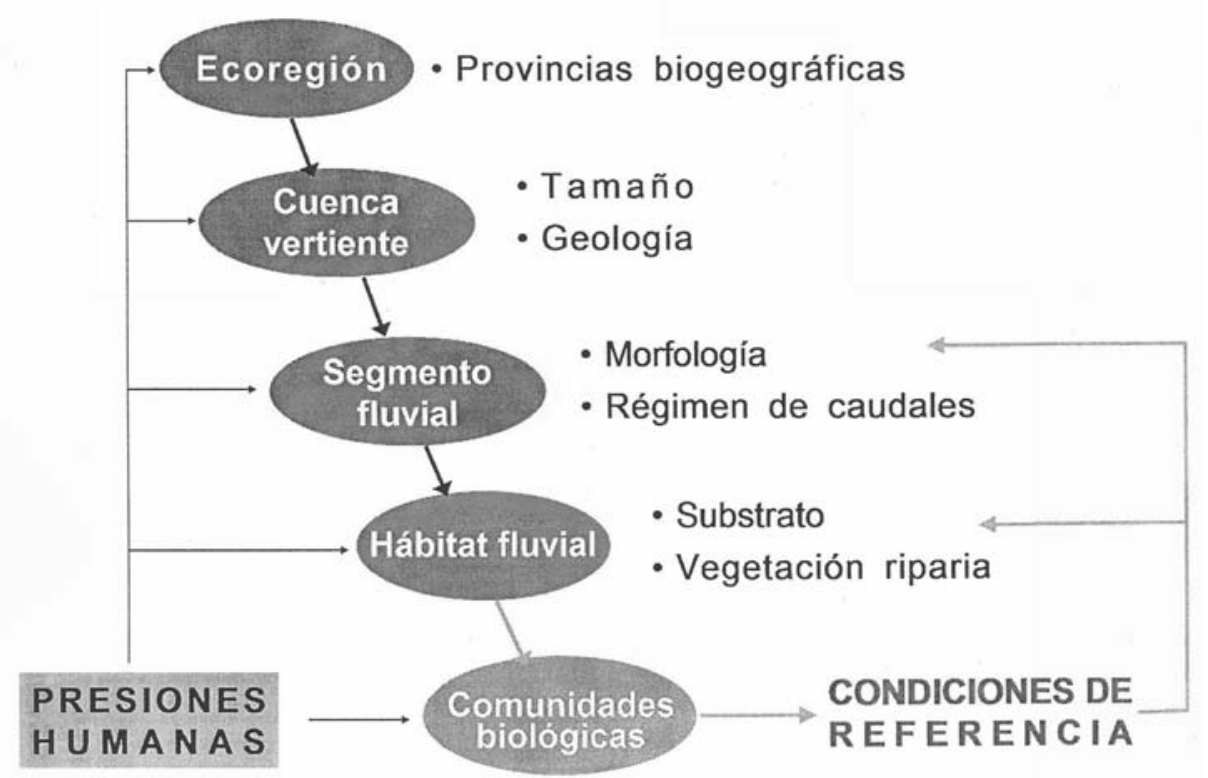

Figura 3. Esquema de caracterización jerárquica de los ríos, atendiendo a la dependencia asimétrica de los factores que determinan sus comunidades biológicas. Hierarchical river characterization scheme, according to the asymetrical dependence of main factors determining biological communities.

de los ríos como fase inicial de la implementación de la Directiva Marco, permite obtener algunas conclusiones, como sigue:

- La mayoría de las tipologías propuestas derivan de análisis estadísticos realizados a partir de una serie de datos disponibles, distintos según las zonas estudiadas, relativos a variables que tienen un nivel de significación hidrológica y ecológica muy diferente. Con ello se obtienen clasificaciones de tipo taxonómico, de escala única, donde se consideran múltiples factores siguiendo un modelo de "caja negra" o gris, que no responde a una ordenación jerárquica previa de dichos factores, basada en el conocimiento científico de su influencia en los ecosistemas fluviales.

- En general, las clasificaciones obtenidas no responden a una interpretación de los procesos físicos e hidrológicos que actúan a diferentes escalas espaciales y temporales en el ecosistema fluvial, ni parten de un planteamiento ecológico de reconocimiento de discontinuidades, o elementos de clasificación, en el contínuo fluvial.
- Los resultados obtenidos hasta la fecha no parecen muy satisfactorios a la hora de poder establecer comparaciones entre los distintos tipos establecidos en relación a sus características físicas, puesto que en muchos casos se definen en base a distintas variables, y por consiguiente, tampoco facilitan la comparación en relación a sus características biológicas.

- La denominación de los grupos de ríos obtenidos alude a las variables utilizadas para su obtención, y no facilita su localización geográfica, agrupando en muchos casos tramos fluviales de diferentes regiones o distritos hidrográficos.

\section{PROPUESTA DE CLASIFICACIÓN JERÁRQUICA DE LOS RÍOS ESPAÑOLES}

La tipología que se propone para la caracterización de los ríos españoles de acuerdo con los objetivos de la Directiva Marco del Agua, parte del reconocimiento de la dependencia jerárquica que mantienen los distintos factores que determinan la estructura y funcionamiento de 


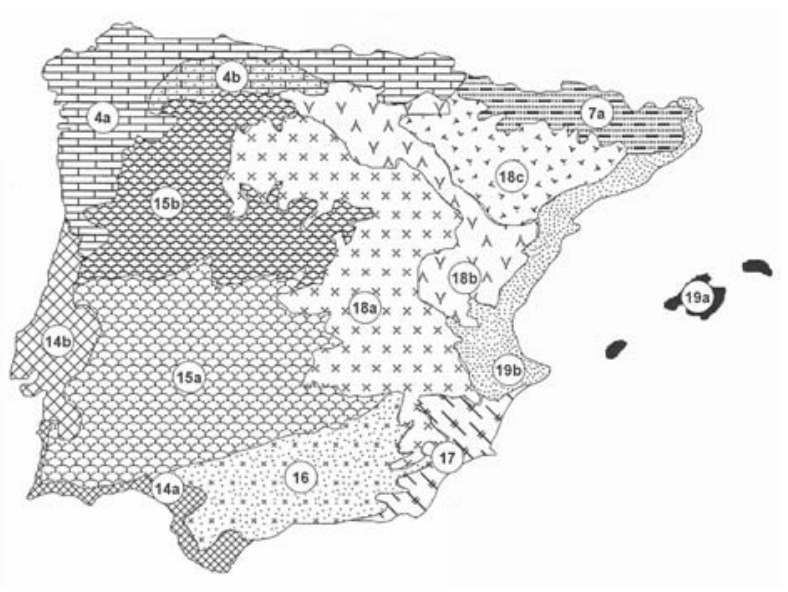

Figura 4. Provincias biogeográficas reconocidas en la Península Ibérica por Rivas Martínez et al. (2002). 4: Provincia Atlántica Europea. 4a: Subprovincia Cántabro-Atlántica; 4b: Subprovincia Orocantábrica. 7: Provincia CevenensePirenaica. 7a: Subprovincia Pirenaica. 14: Provincia LusitanoAndaluza-Litoral. 14a Subprovincia Gaditano-Algarviense. 15: Provincia Mediterránea Ibérica Occidendal. 15a Subprovincia Luso-Extremadurense; 15b Subprovincia Carpetano-Leonesa. 16: Provincia Bética. 17: Provincia Murciano-Almeriense. 18: Provincia Mediterránea Ibérica Central. 18a Subprovincia Castellana. 18b Subprovincia Oroibérica: 18c: Subprovincia Bajo Aragonesa. 19: Provincia Catalana-Provenzal-Balear. 19a Subprovincia Balear; 19b: Subprovincia Catalana-Valenciana. Biogeographic Provinces in the Iberian Peninsula recognized by Rivas Martínez et al. (2002).4: Atlantic European Province. 4a Cantabrian-Atlantic Subprovince; 4b: Orocantabric Subprovince. 7: Cévenense-Pyrenean Province. $7 a$ Pyrenean Subprovince. 14: Coastal Lusitan-Andalusian Province. 14a Gaditan-Algarvian Subprovince. 15: Mediterranean West Iberian Province. 15a Lusitan-Extremadurean Subprovince; 15b: Carpetan-Leonese Subprovince. 16: Betic Province. 17: Murcian-Almeriensian Province. 18: Mediterranean Central Iberian Province. 18a Castillian Subprovince; 18b: Oroiberian Subprovince: 18c: Low Aragonese Subprovince. 19: Balearic-Catalan-Provençal Province. 19a Balearic Subprovince. 19b: Catalan-Valencian Subprovince.

los ecosistemas fluviales, actuando a diferentes escalas espaciales y temporales.

En la figura 3 se ha representado esta dependencia jerárquica de las comunidades biológicas en relación a su hábitat, del hábitat fluvial en relación a las características del tramo de río, y de éste en relación a las de su cuenca vertiente, enmarcada a su vez en una determinada región biogeográfica, basada en los conceptos anteriormente comentados de Frissell et al. (1986); Montgomery \& Buffington (1993), etc.

En condiciones no alteradas por el hombre, o "condiciones de referencia" según la Directiva Marco del Agua, podemos asumir que las características climatológicas y geográficas (biogeográficas) determinan en primer término las condiciones hidrológicas de las cuencas vertientes (Jones, 1999; Dingman, 2002, etc.). En función de la geología, relieve y tamaño de estas últimas, ante dichas condiciones hidrológicas se producen diferentes respuestas o regímenes de caudales, que configuran la morfología de los cauces y determinan la permanencia y fluctuación de las características hidráulicas, siendo éstas a su vez las que generan los respectivos hábitats fluviales para las distintas especies del ecosistema fluvial (Montgomery, 1999; Poff et al., 1997)

La intervención humana altera la dinámica ambiental de los ríos en función de su afección a los distintos factores que influyen en su funcionamiento, a distintas escalas o niveles de control hidrológico y ecológico (Bravard \& Petts, 1993), dando como resultado unas condiciones actuales que pueden diferir notablemente de las consideradas de referencia, no solo en términos biológicos sino también en relación a los elementos hidromorfológicos y físico-químicos considerados en la Directiva Marco.

En esta ocasión, se trata de caracterizar a los distintos tipos de ríos en función de los factores en los que el hombre ha tenido una influencia nula o poco significativa a una escala temporal aproximada de decenas de años, o en los que habiendo modificado sus condiciones, éstas pueden reconstruirse fácilmente a partir de datos o documentación previa a la alteración, siendo posible establecer las condiciones primitivas o consideradas de referencia, según el concepto de "imagen objetivo" o "leitbild" definido por Jungwirth et al. (2002).

El elemento de clasificación propuesto es el "segmento fluvial", definido a partir de la fragmentación natural de los cauces determinada por la confluencia de sus afluentes. La discontinuidad que representa la llegada de los tributarios al cauce principal influye en la dinámica de este último (Benda et al., 2004), y su consideración como criterio para la delimitación de los respectivos segmentos fluviales tiene un claro fundamento hidrológico y ecológico, a la vez 
Tabla 3. Clasificación jerárquica de los hábitats fluviales, incluyendo los factores y criterios de caracterización a diferentes escalas. Hierarchical classification of river habitats, including the factors and characterisation criteria at different spatial scales.

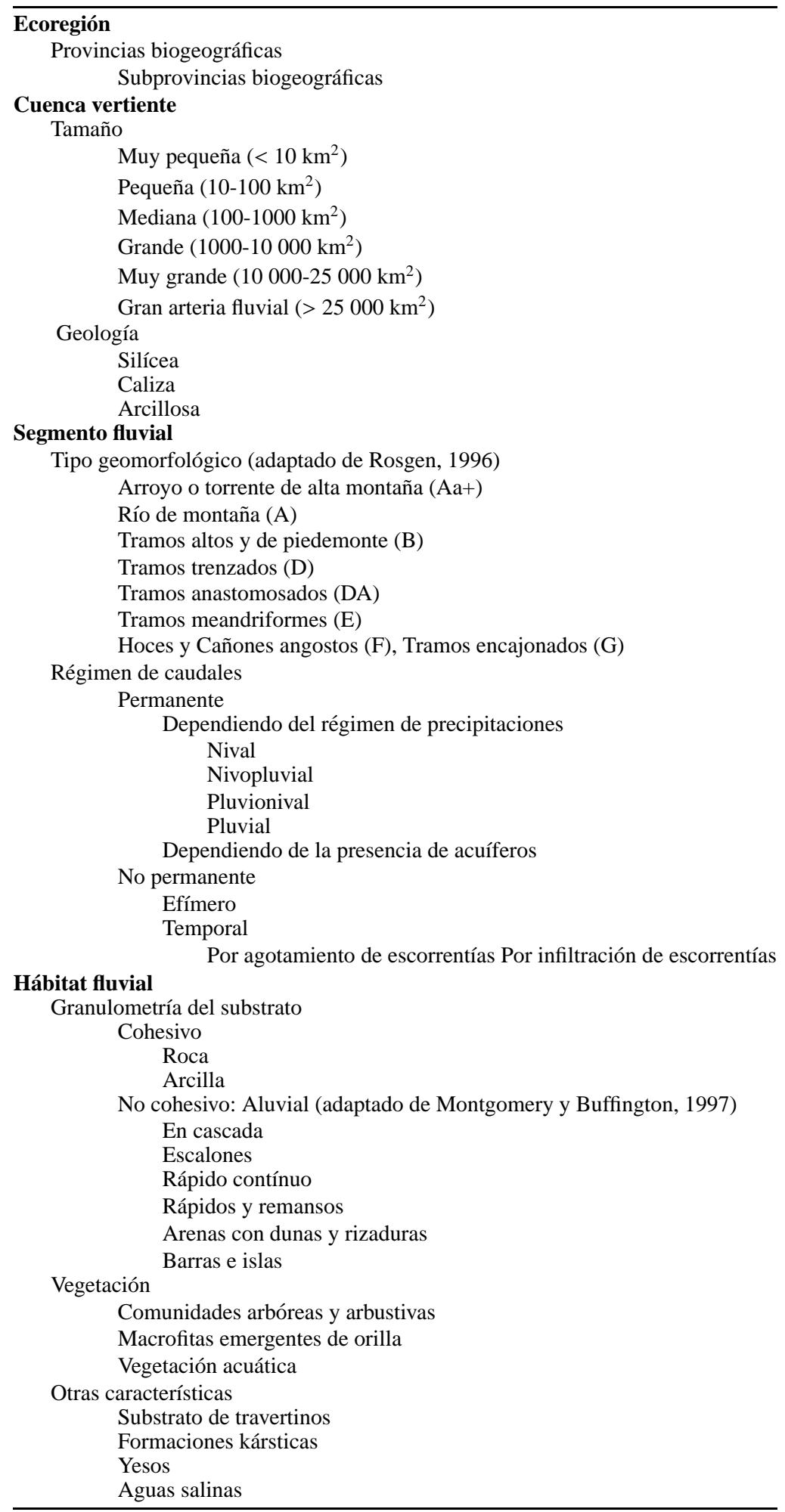


que facilita la diferenciación objetiva de los mismos sobre la cartografía.

Una vez reconocidos los segmentos fluviales, en una disminución de escala espacial (upscaling) se considera la cuenca vertiente al extremo inferior del segmento, y posteriormente la región biogeográfica a la que corresponde dicha cuenca. Dentro de cada segmento, y aumentando la escala espacial (down-scaling), se consideran diferentes unidades funcionales que atienden a discontinuidades físicas, en las que se reconocen a su vez diferentes tipos de hábitats fluviales, sobre los que se pueden analizar las comunidades biológicas.

En la Tabla 3 se presenta la caracterización jerárquica propuesta, incluyendo los diferentes niveles de clasificación y los criterios utilizados para las diferentes escalas espaciales consideradas.

\section{Ecoregión: Provincias biogeográficas}

El reconocimiento de ecoregiones, caracterizadas por factores naturales que actúan a gran escala, es la mejor manera de iniciar la regionalización de un territorio, tratando de definir áreas ecológicamente homogéneas donde llevar a cabo planes de gestión y conservación de los sistemas naturales (Omernik \& Bailey, 1997). Esta consideración de ecoregiones, propuesta en el sistema A de clasificación de la Directiva Marco, está siendo aplicada en varios países, para la elaboración de su respectiva tipología fluvial (Wasson et al., 2002; Brilly et al., 2004).

En España existe una gran experiencia en este tipo de regionalización del territorio español, basada fundamentalmente en los trabajos de Rivas Martínez y colaboradores (Rivas Martínez, 1987; Rivas Martínez et al., 2002), que representa una gran ayuda para integrar la influencia conjunta de factores como el clima, la geología y el relieve en las condiciones hidrológicas de las respectivas cuencas vertientes.

En la figura 4 aparecen representadas las Provincias biogeográficas reconocidas por dichos autores en la Península Ibérica, cuya utilización nos parece de gran interés a la hora de establecer la tipología de los ríos españoles, dado que en su diferenciación se han tenido
Tabla 4. Superficie de cuenca española de los principales ríos de la Península Ibérica. Spanish basin area for the main rivers of the Iberian Peninsula.

\begin{tabular}{lc}
\hline \multicolumn{1}{c}{ Río } & Área vertiente( $\left(\mathbf{k m}^{\mathbf{2}}\right)$ \\
\hline Nervión (País Vasco) & 1764 \\
Ter (Cataluña) & 3011 \\
Guadalhorce (Sur) & 3158 \\
Nalón (Asturias) & 4866 \\
Llobregat (Cataluña) & 4948 \\
Sil (Galicia) & 7982 \\
Miño (Galicia) & 16212 \\
Segura & 19120 \\
Júcar & 42900 \\
Tajo & 55810 \\
Guadiana & 60210 \\
Guadalquivir & 63240 \\
Duero & 78960 \\
Ebro & 85560 \\
\hline
\end{tabular}

en cuenta características termo-pluviométricas, de altitud y pendiente de las laderas, geología y suelos, situación geográfica, etc., muchas de ellas relacionadas con algunos de los descriptores opcionales que incluye el sistema B de clasificación propuesto por la Directiva Marco.

La nomenclatura de estas provincias biogeográficas alude a su ubicación geográfica y facilita considerablemente la localización de los respectivos ríos y segmentos fluviales, hecho que anteriormente ya ha sido considerado de gran interés en la Directiva Europea de Hábitat, donde se ha seguido un esquema de regionalización similar para la gestión y conservación de los respectivos hábitats (Rodwell et al., 1997).

\section{Cuenca vertiente}

Hidrológicamente, el territorio se organiza en cuencas vertientes, cuyas características de funcionamiento y respuesta están condicionadas por la región biogeográfica en la que se enmarcan (Omernik y Griffith, 1991).

Ante similares condiciones climatológicas, la respuesta hidrológica de cada cuenca depende, entre otros factores, de su tamaño, determinando el área de captación de precipitaciones y superficie donde se generan las escorrentías, y de su geología, condicionando las características de infiltración y retención de agua en el interior de la cuenca (Black, 1997). 
Son estas características de tamaño y geología las que propone la Directiva Marco para llevar a cabo la tipología fluvial, y sobre las que proponemos las categorías que aparecen en la Tabla 3.

La Directiva Marco del Agua establece una clase única de cuencas "muy grandes", con superficie mayor de $10000 \mathrm{~km}^{2}$. Dada la amplitud del rango de superficies que podemos encontrar en esta clase dentro del ámbito europeo, y reconociendo diferencias ecológicas que pueden existir atendiendo a la magnitud de los caudales y su predictibilidad (Tabla 4) proponemos la diferenciación de una clase adicional para los tramos bajos de las grandes arterias fluviales, con una superficie vertiente superior a $25000 \mathrm{~km}^{2}$.

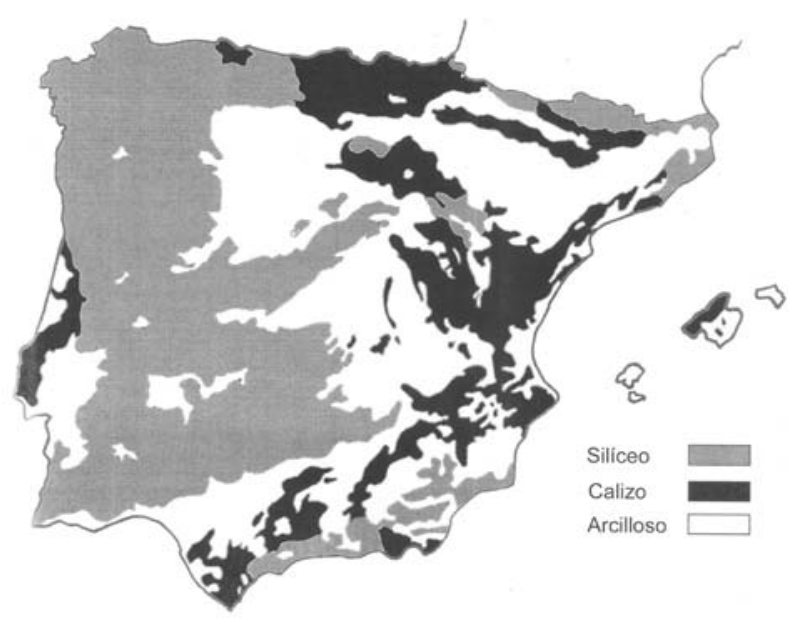

Figura 5. Mapa de las litologías dominantes en la Península Ibérica elaborado por Gutiérrez Elorza (1994). Map of the dominant lithologies in the Iberian Peninsula proposed by Gutiérrez, Elorza (1994).

Para la caracterización de la geología de las cuencas vertientes, y tratando de simplificar la información suministrada por las diferentes cartografías existentes en nuestro país, proponemos la utilización del trabajo de Gutiérrez Elorza (1994), donde se reconocen las categorías silícea, caliza y arcillosa, según se representa en la figura 5.

La Directiva Marco incluye una clase geológica "orgánica" que nosotros no hemos tenido en cuenta, considerando la escasez de su representación en la Península Ibérica. Por el contrario, consideramos que existe una gran superficie cubierta por materiales sedimentarios de tipo arcilloso, recubriendo gran parte de las dos Mesetas y las depresiones de las cuencas mediterráneas, y que es interesante destacar esta categoría no recogida en la Directiva Marco, teniendo en cuenta su influencia en la morfología y tipo de substrato de los cauces.

La adscripción de cada cuenca a una u otra categoría geológica dependerá del porcentaje que exista de cada una de ellas, surgiendo a menudo categorías intermedias a partir de las cuencas de tamaño medio, que pueden quedar caracterizadas a su vez como subclases, dentro de una misma categoría geológica.

\section{Segmento fluvial}

Considerando la confluencia de afluentes como factor de discontinuidad hidrológica en los respectivos cauces principales, proponemos utilizar los segmentos fluviales identificados por la administración pública española (MOP, 1965) para la elaboración de su tipología.

En el trabajo citado, cada río es codificado mediante dígitos, y definido en su longitud y área vertiente en cada uno de los segmentos fluviales que van surgiendo entre confluencias.

Se trata de un estudio básico y de referencia, que ofrece un criterio fisico y "objetivo" de identificación de los segmentos fluviales, fácilmente aplicable sobre cartografía y con fundamento hidrológico y ecológico.

Cada segmento así reconocido es posteriormente caracterizado en función de su régimen de caudales y sus condiciones geomorfológicas, ambos relacionados con la magnitud del flujo circulante y la superficie vertiente, que se ven incrementados sucesivamente, después de la llegada de cada afluente.

En relación a la caracterización del régimen de caudales, y teniendo en cuenta los trabajos de Poff \& Ward (1989) y Olden \& Poff (2003), hemos diferenciado en primer término los regímenes permanentes, con caudal circulante todo el año, de los regímenes no permanentes, conside- 
rando la "temporalidad" de los caudales como uno de los factores principales que condiciona el funcionamiento del ecosistema fluvial. Posteriormente, dentro de cada tipo de régimen hemos considerado su "predictibilidad" y "variabilidad", atendiendo al origen principal de las escorrentías, predecibles pero con oscilaciones estacionales en el caso de responder al régimen de precipitaciones, más homogéneas en el caso de afluencia de aguas subterráneas, y de carácter más torrencial en el caso de los regímenes efímeros, localizados principalmente en las ramblas.

Para la caracterización de las condiciones geomorfológicas de cada segmento fluvial proponemos adaptar la clasificación de Rosgen (1996), considerando los 9 tipos morfológicos que Rosgen reconoce en el primer nivel de su clasificación, basados en la pendiente longitudinal del cauce, el coeficiente de sinuosidad y la forma dominante de la sección transversal (Fig. 2).

En este sentido, y con el fin de poder asignar un tipo morfológico dominante a cada segmento fluvial en base a la cartografía, proponemos asignar los distintos tipos morfológicos en función de los valores de pendiente longitudinal, coeficiente de sinuosidad, y tipo de valle, según su grado de encajonamiento.

\section{Tramo o Sector fluvial}

Finalmente, dentro de cada segmento fluvial se reconocen diferentes sectores o tramos donde las características de substrato, forma de las orillas, estabilidad del cauce, etc., pueden ser diferentes, configurando distintos tipos de hábitats fluviales.

Para la caracterización de los distintos sectores es necesario realizar un reconocimiento de campo en cada segmento fluvial, tras el cual se pueda detallar, en una escala cada vez mayor, las condiciones hidromorfológicas en que se desarrollan las respectivas comunidades biológicas.

La granulometría y organización de las distintas formas del lecho determinan importantes aspectos del hábitat fluvial, estando determinadas por las variables hidráulicas que establecen los caudales circulantes, sobre un cauce no rígido (Hey, 1997). Para su caracterización hemos diferenciado en primer término, siguiendo a Schumm
(1977), el tipo de material cohesivo, de roca o arcilla, del no cohesivo, referido al material transportado y seleccionado por la acción de la corriente (substrato aluvial).

Respecto al substrato no cohesivo, de gravas $\mathrm{y}$ arenas, las distintas formas del lecho que pueden organizarse en cada caso han sido descritas con detalle por Church (1992) y Montgomery \& Buffington (1997). En los sectores de cabecera de alta montaña, el lecho de los ríos y arroyos está constituido por grandes bloques de origen coluvial, que determinan la formación de "cascadas". Hacia aguas abajo, el tamaño medio de estos bloques disminuye, y la corriente es capaz de organizar pequeños "escalones", donde se reconoce una alternancia de pozas y pequeños saltos que afectan respectivamente a toda la sección transversal, todavía de pequeña anchura. Según disminuye la pendiente longitudinal, hacia aguas abajo se forman tramos de "rápidos contínuos", con gravas y cantos rodados, desapareciendo las pequeñas presas organizadas por las piedras de mayor tamaño que constituían los escalones de aguas arriba. Finalmente, hacia aguas abajo, y en función de un tamaño menor de los cantos rodados y abundancia de gravas, se organiza una alternancia de "rápidos y remansos", en la que se reconoce, sobre una anchura de cauce mayor, una variación transversal de las condiciones hidráulicas entre las dos orillas (Fig. 1).

En relación al substrato no cohesivo de arenas, son conocidas las formas del lecho que pueden establecerse en función de la energía hidráulica de la corriente, con "rizaduras" y "dunas" al iniciarse el movimiento de las partículas con una energía hidráulica menor, un "lecho liso" en la fase de transición o movimiento crítico, y la formación de "antidunas" en los casos de elevada potencia hidráulica y movimiento supercrítico (Chang, 1988).

El reconocimiento de los segmentos fluviales con múltiples cauces puede detallarse en función de la forma de las islas, el grado de ramificación de los canales, etc. según se detalla en el trabajo de Thorne (1997).

La vegetación de las riberas, su composición y estructura, cobertura, continuidad, etc., son 
aspectos fundamentales del ecosistema fluvial, que han sido incluidos en la Directiva Marco como elementos hidromorfológicos indicadores del estado ecológico de los ríos (CEN, 2004).

En la aplicación de la Directiva, se trata de valorar las condiciones actuales de la vegetación riparia en los distintos tramos fluviales, en relación a las comunidades que deberían existir en la zona, consideradas como referencia. Por ello proponemos describir la vegetación riparia con el mayor detalle posible, en relación a la formación vegetal dominante en las orillas, en las riberas (en sentido estricto) y en la llanura de inundación, aludiendo a su grado de cobertura, composición específica y continuidad, siguiendo un enfoque similar al propuesto por Winward (2000), con la información de referencia suministrada por Garilleti y Lara (2002).

La aplicación de índices como el QBR (Munné et al.,1998) facilita la valoración comparativa del estado ambiental de las riberas, pero no debería utilizarse en la implementación de la Directiva Marco, donde el objetivo no es tanto establecer una ordenación de "mejor a peor" en función de un valor obtenido con el índice, sino de reconocer la similitud o diferencia entre las condiciones actuales y las de referencia de cada zona, en términos de composición y dinámica de las respectivas formaciones vegetales, que en algunos casos podrían ser muy diferentes del esquema de valoración única implícito en el mencionado índice.

\section{Otras características}

Finalmente, cada tramo o sector fluvial puede particularizarse en función de factores limitantes locales, relativos al tipo de substrato (travertinos, formaciones kársticas, etc.), presencia de geologías específicas (yesos, salinas, aguas termales, etc.) u otros factores que pueden considerarse de interés para caracterizar las condiciones del hábitat en el que se desarrollan las respectivas comunidades biológicas peculiares.

\section{APLICACIÓN DEL ESQUEMA DE CARACTERIZACIÓN JERÁRQUICA PROPUESTO Y CLASIFICACIÓN DE LOS SEGMENTOS FLUVIALES}

El esquema de caracterización jerárquica propuesto puede aplicarse directamente a todo el ámbito de la Península Ibérica, o por grandes regiones hidrográficas (actuales unidades de planificación hidrológica), y está preparado para ser definido mediante programas informáticos (ArcGis 8.3), con los cuales se pueden obtener distintas cartografías, a la escala espacial o de nivel de caracterización deseados.

Se trata de un método de caracterización de toda la red de drenaje considerada sencillo y fácilmente abordable en la actualidad, de tipo arborescente, donde cada segmento fluvial queda caracterizado por su localización geográfica (provincia y subprovincia biogeográfica), atributos de su cuenca vertiente (tamaño y geología) y características hidrológicas (régimen de caudales) y geomorfológicas (tipo de cauce) que determinan sus hábitats fluviales (granulometría del substrato, formas del lecho, orillas, riberas, etc.).

La posibilidad de incorporar de forma general, o individual para un determinado grupo de ríos o segmentos fluviales, cualquier información adicional que se considere de interés para detallar uno o varios de los factores considerados, permite incluir todos los datos complementarios que están disponibles en algunas de las regiones hidrográficas, o pueden estarlo gradualmente en un futuro, en una estructura de datos común a todo el territorio español, siendo un sistema de caracterización abierto y dinámico, en el sentido de aceptar nueva información sin modificar su base conceptual inicial.

Una vez completada la "caracterización" de la red de drenaje se puede proceder a la "clasificación" de los respectivos segmentos fluviales, mediante la ordenación jerárquica de los tramos fluviales considerados y la identificación de tipos de ríos homogéneos. En este caso, son los atributos 
de la cuenca vertiente (tamaño y geología) y tipo de régimen de caudales los factores que pueden facilitar en mayor medida la identificación de tipos o "clases" de ríos, pudiendo surgir dentro de cada clase diferentes "subclases", en función del tipo geomorfológico y substrato del cauce, de su localización geográfica (provincia y subprovincia biogeográfica), o de cualquier otro factor existente en la zona de estudio, atendiendo siempre al mismo esquema común de caracterización y descripción de las características fluviales, con base hidrológica y ecológica.

No todas las posibles combinaciones de los factores utilizados en la caracterización propuesta existen en condiciones naturales, al estar algunos de estos factores correlacionados entre sí (ej. región biogeográfica con geología y régimen de caudales; morfología del segmento fluvial con estructura del substrato, etc.). No obstante, las condiciones actuales pueden diferir de las naturales, y se pueden encontrar combinaciones de factores distintas a las esperadas. En este caso, el esquema de caracterización propuesto ayuda a interpretar las alteraciones debidas a intervenciones humanas, ofreciendo las pautas naturales geomorfológicas e hidrológicas que deben corresponder a cada una de las regiones y tipologías establecidas.

El número de grupos o clases de ríos que puede obtenerse finalmente en cada caso dependerá de la heterogeneidad ecológica de cada región, no debiendo tratar de reducir excesivamente dicho número, teniendo en cuenta que el territorio español es muy diverso en términos ambientales, y que necesariamente hemos de encontrar un número de tipos de ríos diferentes mucho mayor que otras regiones europeas.

Finalmente, este esquema de clasificación de los ríos atendiendo a las principales características físicas que rigen el funcionamiento de los ecosistemas fluviales representa un modelo básico para (1) identificar los tramos de ríos mejor conservados dentro de cada clase establecida; (2) definir, en base a dichos tramos, las condiciones de referencia relativas al tipo de régimen de caudales, morfología del cauce y características de los hábitats que hacen posible el mantenimento de determinadas comunidades biológicas, que corresponden a cada una de las clases de río esta- blecidas; y (3) proponer medidas encaminadas a la mejora y restauración de las mencionadas características físicas, en los tramos fluviales cuyo estado ecológico así lo requiera, atendiendo a las condiciones de referencia establecidas para la tipología de río a la que pertenecen.

\section{BIBLIOGRAFÍA}

ACA (Agencia Catalana de l'Aigua) 2002. Regionalització del sistema fluvial a les Conques internes de Catalunya. Barcelona. 21 pp.

AMOROS, C. et G. E. PETTS. 1993. Bases conceptuelles. En: Hydrosistèmes fluviaux. C.Amoros et G. E. Petts (eds.): 3-17. Chapitre 1. Masson, Paris.

BAILEY, R. G. 1976. Ecoregions of the United States. Map scale 1:7.500.000. U.S. Department of Agriculture, Forest Service. Intermountain Region, Ogden, Utah.

BAILEY, R. G. 1983. Delineation of ecosystem regions. Environmental Management, 7: 365-373.

BAILEY, R. G. 1995. Descriptions of the Ecoregions of the United States ( $2^{\text {nd }}$ Edition). Miscellaneous Publication No. 1391. Map scale 1:7.500.000. U.S. Department of Agriculture, Forest Service. Intermountain Region, Ogden, Utah.

BAILEY, R.G., R. D. PFISTER \& J. A. HENDERSON. 1978. Nature of Land and Resource Classification. A review. Journal of Forestry, 76: 650-655.

BENDA, L., N. L. POFF, D. MILLER, T. DUNNE, G. REEVES, G. PESS \& M. POLLOCK. 2004. The network dynamics hypothesis: How channel networks structure riverine habitats. BioScience, 54: 413-427.

BLACK, P. E. 1997. Watershed Functions. J. Am. Water Res. Assoc., 33: 1-11.

BONADA, N. N. PRAT, A. MUNNÉ, M. RIERADEVALL, J. ALBA-TERCEDOR, M. ÁLVAREZ, J. AVILÉS, J. CASAS, P. JÁIMEZ-CUÉLLAR, A. MELLADO, G. MOYÁ, I. PARDO, S. ROBLES, G. RAMÓN, M.L. SUÁREZ, M. TORO, M.R. VIDAL-ABARCA, S. VIVAS y C. ZAMORAMUÑOZ. 2002 Ensayo de una tipología de las cuencas mediterráneas del proyecto GUADALMED siguiendo las directrices de la directiva marco del agua. Limnetica, 21 (3-4): 77-98

BORMAN, F. H. \& G. E. LIKENS. 1981. Pattern and Process in a Forested Ecosystem. SpringerVerlag, New York. 253 pp. 
BRAVARD, J. P. et G. E. PETTS. 1993. Interférences avec les interventions humaines. In : Hydrosistèmes fluviaux. C. Amoros et G. E. Petts (eds.): 233-253. Masson, Paris.

BRICE, J. C. 1975. Air photo interpretation of the form and behaviour of alluvial rivers. Final report to the US Army Research Office.

BRILLY, M., L. GLOBEVNIK \& A. VIDMAR. 2004. Estimation of ecohydrographical regions in Slovenia. 3rd European Conference on River Restoration, Zagreb, Croatia.

CEDEX 2004. Caracterización de los tipos de ríos y lagos. Versión 1.0. Centro de Estudios Hidrográficos, Ministerio de Fomento, Madrid. 204 pp.

CEN (Comité Européen de Normalisation). 2004. Water quality- Guidance standard for assessing the hydromorphological features of rivers. Final draft prEN 14614, European Standard. 21 pp.

CHANG, H. H. 1988. Fluvial Processes in River Engineering. John Wiley \& sons, New York. 432 pp.

CHURCH, M. 1992. Channel Morphology and Typology. En: River Handbook, Vol. I. P. Calow \& G. E. Petts (eds.): 126-143. Blackwell Scientific Publication, Oxford, U.K.

COMISIÓNEUROPEA. 2000. Directive 2000/60/EC of the European Parliament and of the Council of 23 October 2000 establishing a framework for Community action in the field of water policy. OJEC (Official Journal of the European Communities) 22.12.2000. 72 pp.

CUMMINS, K. W. 1974. Structure and function of stream ecosystems. BioScience, 24: 631-641.

DETENBECK, N. E., C. M. ELONEN \& D. L. TAYLOR. 2004. Region, Landscape and Scale Effects on Lake Superior Tributary Water Quality. J. Am. Water Res. Assoc., 4: 705-720.

DINGMAN, S. L. 2002. Physical Hydrology. Prentice Hall, New Jersey, USA. 646 pp.

DOPPELT, B., M. SCURLOCK, C. FRISSELL \& J. KARR. 1993. Entering the Watershed. Island Press, Washington D.C. 462 pp.

DOUGLASS, J. E. \& M. D. HOOVER. 1988. History of Coweeta. En: Forest Hydrology and Ecology at Coweeta. W. T. Swank \& D. A. Crossley (eds.): 17-31. Springer-Verlag, New York.

DOYLE, M. W., D. E. MILLER \& J. M. HARBOR. 1999. Should River Restoration be based on classification schemes or Process Models? Insights from the history of Geomorphology. ASCE
International Conference on Water Resources Engineering, Seattle, Washington.

DRISCOLL, R. S., D. R. BETTERS \& H. D. PARKER. 1978. Land Classification through remote sensing. Techniques and Tools. Journal of Forestry, 76: 656-661.

FRISSELL, C., W. J. LISS, C. E. WARREN \& M. D. HURLEY. 1986. A Hierarchical framework for Stream Habitat Classification: Viewing Streams in a Watershed context. Environmental Management, 10: 199-214.

GOODWIN, C. N. 1999. Fluvial Classification: Neanderthal Necessity or Needless Normalcy. In: Wildland Hydrology. D. S. Olson \& J. P. Potyondy (eds.): 229-236. American Water Resources Association, Virginia.

GRIFFITH, G. E., J. M. OMERNIK \& A. J. WOODS. 1999. Ecoregions, watersheds, basins and HUCs: How state and federal agencies frame water quality. Journal of Soil and Water Conservation, 54(4): 666-677.

GUTIÉRREZ ELORZA, M. 1994. Introducción a la Geomorfología Española. En: Geomorfología de España. M. Gutiérrez Elorza (ed.): 1-24. Ed. Rueda, Madrid.

HAWKES, H. A. 1975. River zonation and classification. In: River Ecology. B. A. Whitton (ed.): 312374. Blackwell, London, U.K.

HAWKINS, C. P. \& R. H. NORRIS (eds.). 2000. Landscape Classifications: Aquatic Biota and Bioassessments. J. North Am. Benthol. Assoc., 19: 367-571.

HAWKINS, C. P., R. H. NORRIS, J. GERRITSEN, R. M. HUGHES, S. K. JACKSON, R. K. JOHNSON \& R. J. STEVENESON. 2000. Evaluation of the use of landscape classifications for the prediction of freshwater biota: synthesis and recommendations. J. North Am. Benthol. Assoc., 19: 541-556.

HEY, R. D. 1997. Stable River Morphology. In: Applied Fluvial Geomorpholgy for River Engineering and Management. R. Thorne, R. D. Hey \& M. D. Newson (eds.): 223-236. John Wiley \& sons, Chicheter, U.K.

HOLMES, N. T. H. 1989. British rivers: A working classification. British Wildlife, 1: 20-36.

HYNES, H .B. N. 1975. The stream and its valley. Verh. Internat. Verein. Limnol., 19: 1-15.

HUET, M. 1954. Biologie, profils en long et en travers des eaux courantes. Bulletin Francais de Pisciculture, $175: 41-53$. 
ILLIES, J. et L. BOTOSANEANU. 1963. Problémes et méthodes de la classification et de la zonation écologique des eaux courantes considerées surtout du point de vue faunistique. Mitt. Internat. Verein. Theor. Angew. Limnol., 12: 1-57.

JONES, J. A. A. 1999. Global Hydrology. Longman, Essex, England. 399 pp.

JUNGWIRTH, M., S. MUHAR \& S. SCHMUTZ. 2002. Re-establishing and assessing ecological integrity in riverine landscapes. Freshwat. Biol., 47: 867-887.

KONDOLF, G. M. 1995. Geomorphological stream channel classification in aquatic habitat restoration : uses and limitations. Aquatic Conservation: Marine and Freshwater Ecosystems, 5: 127-141.

KONDOLF, G. M., D. R. MONTGOMERY, H. PIÉGAY \& L. SCHMITT. 2003. Geomorphic Classification of Rivers and Streams. In: Tools in Fluvial Geomorphology, G. M. Kondolf \& H. Piégay (eds.): 171-204. John Wiley \& sons, Chichester, U.K.

LARA, F., R. GARILLETI y J. A. CALLEJA. 2004. La vegetación de ribera de la mitad norte española. CEDEX Monografías, 81. Madrid. $536 \mathrm{pp}$.

LEOPOLD, L. B. \& M. G. WOLMAN. 1957. River Channel Patterns- Braided, Meandering and Straight. US Geological Survey Professional Paper 282(B): 39-85.

LIKENS, G. E., F. H. BORMAN, R. S. PIERCE, J. S. EATON \& N. M. JOHNSON. 1977. BioGeo-Chemistry of a Forested Ecosystem. SpringerVerlag, New York. 146 pp.

LOTSPEICH, F. B. 1980. Watersheds as the basic ecosystem: This conceptual framework provides a basis for natural classification system. Water Res. Bull., 16: 581-586.

LOTSPEICH, F. B. \& W. S. PLATTS. 1982. An Integrated Land-Aquatic Classification System. North American Journal of Fisheries Management, 2: 138-149.

MEYBECK. M. \& R. HELMER. 1989. The quality of rivers: from pristine stage to global pollution. $\mathrm{Pa}$ laeography, Palaeoclimatology, Palaeoecology (Global and Planetary Change Section), 75: 283-309.

MONTGOMERY, D. R. \& J. M. BUFFINGTON. 1993. Channel classification, prediction of channel response and assessment of channel condition. TFW-SH10-93-002, Timber, Fish and Wildlife Agreement, Department of Natural Resources, Olympia, Washington. 84 pp.
MONTGOMERY, D. R. \& J. M. BUFFINGTON. 1997. Channel-reach morphology in mountain drainage basins. Geological Society of America Bulletin, 109: 596-611.

MONTGOMERY,D. R. 1999. Process Domains and the River Continuum. J. Am. Water Res. Assoc., 35: 397-410.

MOP (Ministerio de Obras Públicas). 1965. Datos físicos de las corrientes clasificadas por el C.E.H. (Centro de Estudios Hidrográficos). Sección de Planificación. Madrid.

MUNNÉ, A., C. SOLÁ y N. PRAT. 1998. Un índice rápido para la evaluación de la calidad de los ecosistemas de ribera. Tecnología del Agua, 175: 20-37.

NAIMAN, R. J. 1998. Biotic Stream Classification. In: River Ecology and Management. R. J. Naiman \& R. E. Bilby (eds.): 97-119. Springer-Verlag, New York.

NAIMAN, R. J., D. G. LONZARICH, T. J. BEECHIE \& S. C. RALPH. 1992. General principles of classification and the assessment of conservation potential in rivers. In: River conservation and management. P. Boon, P. Calow \& G. Petts (eds.): 93-123. John Wiley \& Sons, Chichester, U. K.

NELSON, DE V., G. A. HARRIS \& T. E. HAMILTON. 1978. Land and Resource Classification. Who Cares? Journal of Forestry, 76: 644-646.

OLDEN, J. A. \& N. L. POFF. 2003. Redundancy and the choice of hydrologic indices for characterizing streamflow regimes. River Research and Applications, 19: 101-121.

OLLERO, A., T. ECHEVARRÍA, M. SÁNCHEZ, V. AURÍA, D. BALLARÍN y D. MORA. 2003. Metodología para la tipificación hidromorfológica de los cursos fluviales de Aragón en aplicación de la Directiva Marco de Aguas (2999/60/CE). Geographicalia, 44: 7-25.

OMERNIK, J. M. 1987. Ecoregions of the Conterminous United States. Annals of the Association of American Geographers, 77: 118-125.

OMERNIK, J. M. 1995. Ecoregions: a spatial framework for environmental management. In: Biological Assessment and Criteria. Tools for water resource planning and decision making. W.S. Davis y T.P. Simon (eds.): 49-62. Lewis Pub., Florida.

OMERNIK, J. M. y R. G. BAILEY. 1997. Distinguishing between watersheds and ecoregions. $J$. Am. Water Res. Assoc., 33: 935-949.

OMERNIK, J. M. \& G. E. GRIFFITH. 1991. Ecological regions versus hydrologic units: Frameworks 
for managing water quality. Journal of Soil and Water Conservation, 46: 334-340.

PLATTS, W. S. 1974. Geomorphic and aquatic conditions influencing salmonids and stream classification- with application to ecosystem classification. Intermountain Forest and Range Experiment Station, Forest Service, U.S.D.A., Boise, Idaho, USA.

POFF, N. L. y J. V. WARD. 1989. Implications of Streamflow Variability and Predictibility for Lotic Community Structure: A regional analysis of streamflow patterns. Can. J. Fish. Aquat. Sci., 46: 1805-1818.

POFF, N. L. \& J. V. WARD. 1990. The physical habitat template of lotic systems: Recovery in the context of historical pattern of spatio-temporal heterogeneity. Environmental Management, 14: 629-646.

POFF, N. L., J. D. ALLAN, M. B. BAIN, J. R. KARR, K. L. PRESTEGAARD, B. D. RICHTER, R. E. SPARKS \& J. C. STROMBERG. 1997. The Natural Flow Regime. BioScience, 47(11): 769-784.

REFCOND. 2003. Guidance on establishing reference conditions and ecological status class boundaries for inland surface waters. EU Common Implementation Strategy for the Water Framework Directive. $86 \mathrm{pp}$.

RIVAS MARTÍNEZ, S. 1987. Mapa de series de vegetación de España. 1:400.000. Publ. ICONA, Serie Técnica, Madrid.

RIVAS MARTÍNEZ, S., T. E. DÍAZ, F. FERNÁNDEZ-GONZÁLEZ, J. IZCO, J. LOIDI, M. LOUSA \& A. PENAS. 2002. Vascular Plant Communities of Spain and Portugal. Itinera Geobotánica, 15(1-2).

ROBERTSON, D. M. \& D. A. SAAD. 2003. Environmental Water Quality Zones for Streams: A Regional Classification Scheme. Environmental Management, 31(5): 581-602.

RODWELL, J. S., L. MUCINA, S. PIGNATTI, J. H. J. SCHAMINÉE \& M. CHYTRÝ. 1997. European
Vegetation survey: The context of the case studies. Folia Geobot. Phytotax., 32: 113-115.

ROSGEN, D. 1994. A classification of natural rivers. Catena, 22: 169-199.

ROSGEN, D. 1996. Applied River Morphology. Wildland Hydrology, Pagosa Springs, Colorado, USA.

SCHUMM, S. A. 1977. The Fluvial System. John Wiley \& sons, New York. 338 pp.

THORNE, C. R. 1997. Channel types and morphological classification. In: Applied Fluvial geomorphology for River engineering and management. C.R. Thorne, R.D. Hey \& M.D. Newson (eds.): 175-222. John Wiley \& sons, Chichester, U. K.

VANNOTE, R. L., G. W. MINSHALL, K. W. CUMMINS, J. R. SEDELL \& C. E. CUSHING. 1980. The river continuum concept. Can. J. Fish. Aquat. Sci., 37: 130-137.

WARREN, C. E. 1979. Toward classification and rationale for watershed management and stream protection. EPA-600/3-79-059. U.S. Environmental Protection Agency, Research Laboratory, Corvallis, Oregon, USA.

WASSON, J. G., A. CHANDESRIS, H. PELLA et L. BLANC. 2002. Les hydro-écorégions de France metropolitane. Approche régionale de la typologie des eaux courantes et éléments pour la définition des peuplements de référence d'invertébrés. Programe de Recherche CEMAGREF, Lyon, Francia. $91 \mathrm{pp}$.

WILLIAMS, J. E., C. A. WOOD \& M. P. DOMBECK (eds.). 1997. Watershed Restoration: Principles and Practices. American Fisheries Society, Bethesda, USA. 561 pp.

WINWARD, A. H. 2000. Monitoring the Vegetation Resources in Riparian Areas. USDA Forest Service, GTR RMRS-GTR-47, Rocky Mountain Research Station, Colorado. 49 pp.

WRIGHT, J. F., P. D. ARMITAGE, M. T. FURSE \& D. MOSS. 1989. Prediction of invertebrate communities using stream measurements. Regulated Rivers: Research and Management, 4: 147-155. 
